This document is confidential and is proprietary to the American Chemical Society and its authors. Do not copy or disclose without written permission. If you have received this item in error, notify the sender and delete all copies.

\title{
Interfacial tension for various organic-water systems and study of the effect of solute concentration and temperature
}

\begin{tabular}{|r|l|}
\hline Journal: & Journal of Chemical \& Engineering Data \\
\hline Manuscript ID & je-2016-00703d.R3 \\
\hline Manuscript Type: & Article \\
\hline Date Submitted by the Author: & O9-Mar-2017 \\
\hline Complete List of Authors: & $\begin{array}{l}\text { Shahid, Muhammad Zubair; Universiti Teknologi PETRONAS, Chemical } \\
\text { Engineering } \\
\text { Usman, Muhammad; Institute of Chemical Engineering and Technology, } \\
\text { University of the Punjab, } \\
\text { Akram, Muhammad Sarfraz; COMSATS IIT , Department of Chemical } \\
\text { Engineering } \\
\text { Khawaja, Shahzad; University of the Punjab, Institute of Chemical } \\
\text { Engineering and Technology } \\
\text { Afzal, Waheed; University of Aberdeen, UK, Chemical Engineering }\end{array}$ \\
\hline
\end{tabular}

SCHOLARONE

Manuscripts 


\title{
Interfacial tension for various organic-water systems and study of the effect of solute
} concentration and temperature

\author{
Muhammad Zubair Shahid ${ }^{1}$, Muhammad Rashid Usman ${ }^{1}$, Muhammad Sarfraz Akram², Shahzad \\ Younus Khawaja ${ }^{1}$, Waheed Afzal ${ }^{1,3 *}$ \\ ${ }^{1}$ Institute of Chemical Engineering and Technology, University of the Punjab, Lahore 54590, Pakistan \\ ${ }^{2}$ Department of Chemical Engineering, COMSATS Institute of Information Technology, Lahore 54000, Pakistan \\ ${ }^{3}$ School of Engineering, University of Aberdeen, Aberdeen AB24 3UE, Scotland, United Kingdom
}

\begin{abstract}
Interfacial tension is an important thermophysical property for the operations involving multi fluid-phases such as liquid-liquid extraction. This work aims at presenting a rapid experimental method and initial interfacial tension data of 10 organic compounds and water binary systems. The organic compounds include n-butyl acetate, cyclohexanol, cyclohexanone, diethyl ether, ethyl acetate, methyl ethyl ketone, methylcyclohexane, 1-octanol, and toluene. The effect of temperature on the initial interfacial tension has also been studied. These systems are chosen to cover a wide range of interfacial tension (1-51 mN/m). Comparisons between the data sets from this work and those from the literature, whenever available, show generally very good agreement. This work also presents new data of initial interfacial tension for several ternary systems with 4 organic compounds and water with varying quantities of propionic acid ( 0 to 0.25 mass fraction) at ambient conditions; the organic compounds include n-butyl acetate, cyclohexanone, 1-octanol, and toluene. The results show that the impact of propionic acid concentration as solute in water is large, especially at the higher solute concentrations. The initial and final (mutually saturated phases) interfacial tensions are found in agreement for different immiscible binary systems studied in this work. The method presented may be used as a rapid way of finding interfacial tensions.
\end{abstract}

\section{Keywords}

Initial interfacial tension, drop weight method, liquid-liquid extraction, propionic acid, waterorganic system.

*Corresponding author: Waheed Afzal, School of Engineering, University of Aberdeen, Kings College, Fraser Noble Building Aberdeen AB24 3UE, Scotland, United Kingdom. Tel: +44 1224 272526 |Fax: +44 (0) 1224272497 |E-mail: waheed@abdn.ac.uk 


\section{Introduction}

Interfacial tension is defined as the change in Gibbs free energy per unit change in interfacial area for the two immiscible phases such as liquid-liquid or gas-liquid ${ }^{1}$. Interfacial tension in liquid-liquid systems plays an essential role in the analysis and design of the equipment used in liquid-liquid contacting systems such as liquid-liquid extraction. It greatly affects the hydrodynamics and mass transfer behavior of a liquid-liquid extractor. For example, a low interfacial tension system is characterized by small size dispersed phase droplets that may increase the dispersed phase holdup as well as the mass transfer efficiency ${ }^{2}$. On the other hand, low interfacial tension may lead to foaming in certain cases that may or may not be desired. Interfacial tension of a liquid-liquid system may vary significantly in the presence of a solute that influences the working and design of the liquid-liquid extractor. Along the length of a typical column type extractor, solute concentration varies in both the phases; this variation results in a variable interfacial tension along the height of the column. A better understanding of the relationship between solute concentration and interfacial tension may therefore be essential for the operation and design of a liquid-liquid extractor ${ }^{2,3}$. Interfacial tension may vary with temperature that may also affect the performance of the extractor.

Interfacial tension for binary systems such as water-organic systems has been studied by a number of researchers ${ }^{4-26}$. Demond and Lindner ${ }^{1}$ and Freitas et al. ${ }^{27}$ have presented excellent reviews in the literature. The interfacial data are generally determined at near-ambient conditions; the effect of temperature on the interfacial tension of a system has rarely been reported in the literature. Also, the data of multicomponent systems, i.e., in the presence of a solute are scarce ${ }^{13}$.

For sparingly-soluble biphasic systems, the initial interfacial tension (without reaching equilibrium) and equilibrium interfacial tension are same; often different researchers do not explicitly mention if both the liquids are saturated with each other before the measurements or not. In a typical measurement, interfacial tension (and densities) of the mutually saturated phases (equilibrated phases) should be reported. Calculation of interfacial tension often requires densities of saturated phases. Initial interfacial tension can be different from final or equilibrium interfacial tension or simply interfacial tension as it does not require saturation of the phases; it also uses the densities of pure components for the calculation purposes. No water would be dissolved in the organic phase and vice versa. During the measurement, the water droplet is exposed to the organic phase which could lead to a gradual ongoing saturation in both the phases. The same holds for the ternary systems studied. Because all the experiments are concluded in a relatively shorter time span in our work, therefore it is expected that amounts of solutes transferred between two phases are negligibly small. 
Here, the interfacial tension is based on virtually pure component interactions and not on the interactions between the mutually saturated phases. This type of interfacial tension (initial interfacial tension) may be important in the startups and in the sections near the inlet and outlet of an extraction column.

In the present study, initial interfacial tensions of ten binary aqueous-organic systems are experimentally determined and the variations in initial interfacial tensions of the selected aqueous-organic systems with the variation in temperature and propionic acid (solute) concentration in the aqueous solution are studied. Apparently, no other initial interfacial tension data seem to be available for the systems studied in the present work. Industrially propionic acid is produced via hydrocarboxylation of ethylene in the presence of water using nickel carbonyl as the catalyst. Numerous industrial applications of propionic acid involve water that warrants study of its aqueous ternary systems with selected organic compounds.

While we selected propionic acid as a representative carboxylic acid due to the fact that it often found in aqueous forms in a number of industrial and natural processes, the main purpose of selecting it is the fact that the systems studied in this work are not reported earlier. Furthermore, we have covered a large range of interfacial tension by selecting a variety of binary and ternary systems including those with propionic acid. We are aware of the fact that the propionic acid will likely change intermolecular interaction perhaps due to $\mathrm{pH}$ change of the water phase that can be one of the reasons of the changes in interfacial tension with concentration, nevertheless, this work reports useful quantitative data of these changes. We studied a fairly large range of propionic acid concentrations -starting from very low to fairly high- to be consistent with our earlier objective, i.e., covering a wide range of interfacial tension. Because propionic acid is often encountered in its aqueous solutions therefore we find it relevant and useful.

\section{Experimental}

The drop weight method ${ }^{1,4,5,8}$ is used to determine the initial interfacial tension. In the drop weight method, the weight of the heavier phase drop becomes equal to the interfacial force that occurs at the interface of the phases involved and the weight of the drop is a measure of the interfacial tension. The drop of one liquid phase formed by tubing detaches and falls into the body of the other liquid phase. Measuring the weight of the drop and the tubing radius provide the magnitude of the interfacial tension present between the two liquid phases. As the detachment is not perfect therefore a correction factor is required to balance the gravity and the interfacial tension forces. 
By initial interfacial tension, as discussed earlier, we mean that the phases are not mutually saturated or equilibrated before the actual measurement. All efforts are made to make sure that the measurements are conducted at a slow pace to avoid experimenter's influence; the measurements are concluded during the initial phase of equilibration between two phases.

Fig. 1 shows a purpose-built equipment constructed, calibrated, and used in this work. It mainly consisted of a cylindrical glass cell and a pre-calibrated and graduated $1.0 \mathrm{~cm}^{3}$ syringe fitted with a removable stainless steel needle (Rudolph Research Analytical, product number P23897) with clean-cut tip perpendicular to the bore of known inside and outside diameters, verified using a screw gauge with least count $\pm 0.01 \mathrm{~mm}$. The inside diameter of the needle was $1.06 \mathrm{~mm}$ while the outside diameter was $1.60 \mathrm{~mm}$. The internal diameter was verified by a set of stainless-steel wires with known, gradually-increasing and uniform diameter. The uncertainty associated with the representative needle diameter and that associated with the droplet volume show acceptable influence (within the standard deviation of quadruplicates) on the interfacial tension data reported in this work. In order to maintain the temperature of measurement, the cylindrical cell was placed inside the water thermostat. The temperature was maintained with $\pm 0.25 \mathrm{~K}$, measured by a mercury thermometer with least count within temperature stability of $0.25 \mathrm{~K}$. A purpose-built screw assembly was provided to drive the syringe plunger by which it was possible to move the syringe plunger at a sufficiently slow pace so that the correct size of the drop was produced.

In a typical procedure, the cell was partially filled with the lighter phase (organic phase) and the heavier phase (aqueous phase) was allowed to drop from the syringe. Every care was taken to avoid vibrations which could lead to the formation of a premature drop. Several of these drops were formed and volume of one drop was obtained by taking the ratio of the total volume displaced in the syringe to the total number of drops formed. The procedure for each experimental data was repeated at least five times (but often 8-10 times) and the average value of the drop volume was obtained. The relative standard deviation of high interfacial tension $(>15)$ was less than $2 \%$ whereas for low interfacial tension was rarely as high as $4 \%$ but often less than $3 \%$.

Interfacial tensions of 10 binary water-organic systems were studied near room temperature. The experiments for studying the effect of temperature on interfacial tension were conducted, for five binary water-organic systems, between $283.15 \mathrm{~K}$ and $333.15 \mathrm{~K}$. For observing the effect of solute concentration, propionic acid concentration $\left(w_{\mathrm{HPr}}\right)$ in water was varied between 0 to 0.25 weight fraction and interfacial tensions of 4 water-organic systems were studied. All the experiments were conducted at ambient pressure. The information such as purity, water content, and the supplier regarding the chemicals used in the present work is given in Table 1. All the 
chemicals were used as received without any further purification. All solutions are prepared gravimetrically using a mass balance with $\pm 10^{-4} \mathrm{~g}$.

\section{Results and Discussion}

The following expression was used for calculating the interfacial tension of a given system after experimentally finding the drop volume of the system:

$$
\gamma_{0}=\frac{\left(\rho_{h}-\rho_{l}\right) \cdot g \cdot V_{d}}{2 \cdot \pi \cdot r_{C} \cdot F}
$$

where, $\gamma_{0}$ is initial interfacial tension, $\rho_{h}$ is density of heavy phase, $\rho_{l}$ is density of light phase, $g$ is acceleration due to gravity, $V_{d}$ is volume of a single drop, $r_{C}$ is radius of the tip of the needle, and $F$ is correction factor mentioned above that accounts for the drop portion that does not fall with the drop. It is important to mention that the representative radius used in Eqs 1-4 for the needle needs special attention ${ }^{28}$. Often the studies do not explicitly pay attention to the fact that if they have to use outer radius, the needle material must be completely wettable with all of the liquids studied and by introducing some roughness ${ }^{28}$ in the tip of needle cut perpendicularly. It may be useful to pay attention to the hydrophobicity (and hydrophilicity) of the needle-tip surface to determine the characteristic radius. Ideally outer radius should be used but for completely wetted tip-cut surface. We conducted a series of experiments with different systems reported in the literature, and found out that for a clean- and perpendicular- cut needle, the most suitable characteristic radius is average of internal and external radii.

The densities used in Eq. 1 were not that of mutually saturated phases, but are those of pure components and pure aqueous solutions of propionic acid. For any required temperature, the densities for 1-octanol, n-butyl acetate, ethyl acetate, diethyl ether, and propionic acid aqueous solutions at different mole fractions were obtained by curve fitting the literature data while densities of toluene, methylcyclohexane, cyclohexanone, and cyclohexanol were measured using the Rudolph Research Analytical vibrating tube automatic density meter. As part of our experimental method, the radius of tip $\left(r_{C}\right)$ was taken as the average of outer and inner radius of the needle, as explained earlier. The following equations were used for calculating the correction factor $F^{28,29}$; different empirical correlations are recommended for different sizes of capillary needles and drops:

$$
F=f\left(\frac{r_{C}}{\sqrt[3]{V_{d}}}\right)=0.998-1.073\left(\frac{r_{C}}{\sqrt[3]{V_{d}}}\right), \quad 0.06<\frac{r_{C}}{\sqrt[3]{V_{d}}}<0.17
$$




$$
\begin{aligned}
& F=f\left(\frac{r_{C}}{\sqrt[3]{V_{d}}}\right)=0.992-1.2355\left(\frac{r_{C}}{\sqrt[3]{V_{d}}}\right)+1.161\left(\frac{r_{C}}{\sqrt[3]{V_{d}}}\right)^{2}, \quad 0.17<\frac{r_{C}}{\sqrt[3]{V_{d}}}<0.30 \\
& F=f\left(\frac{r_{C}}{\sqrt[3]{V_{d}}}\right)=\left(2 \times \pi\left(0.14782+0.27896\left(\frac{r_{C}}{\sqrt[3]{V_{d}}}\right)-0.1662\left(\frac{r_{C}}{\sqrt[3]{V_{d}}}\right)^{2}\right)\right)^{-1}, \quad 0.3<\frac{r_{C}}{\sqrt[3]{V_{d}}}<1.2
\end{aligned}
$$

Because of the empirical nature of factor $F$ in Eq 1, the reader will find different correlations in the literature for the correction factor, we particularly found out that the equation compiled by Drelich et al. ${ }^{30}$ is only applicable to a limited range $\left(r_{C} / V_{d}^{1 / 3} \geq 0.30\right)$, contrary to their description that suggests if it is a general case, which is somewhat misleading.

\section{Binary initial interfacial tensions}

Initial interfacial tensions of 10 binary organic-water systems are measured and reported in Table 2 along with the literature values for these systems where the literature values are those of mutually saturated phases. The initial interfacial tensions are expected to be different from the interfacial tensions because of the different densities used in Eq. 1 in the two cases, different interfacial associations in mutually saturated phases and initially pure phases, and the occurrence of any chemical reaction such as hydrolysis in the case of esters. Table 1 shows that apart from methylcyclohexane the initial interfacial tensions measured are fairly close to the literature values of interfacial tensions though it is expected that the measured initial interfacial tensions for substances that have relatively high mutual solubility with water are expected to have a relatively different value than the interfacial tensions measured when the phases are mutually saturated. On the other hand, the substances which have negligible solubility in water are expected to have virtually the same values of the two types of interfacial tensions. The cases for methylcyclohexane and cyclohexanol need some explanation. In the present work, the initial interfacial tension of methylcyclohexane with water is measured at $293.15 \mathrm{~K}$ as $51.40 \mathrm{mN} / \mathrm{m}$ while the literature ${ }^{5}$ value is $41.9 \mathrm{mN} / \mathrm{m}$ at $298.15 \mathrm{~K}$. As suggested by Apostoluk and Drzymala $^{31}$, the literature value of $41.9 \mathrm{mN} / \mathrm{m}$ is not possible and should be between $50-51$ $\mathrm{mN} / \mathrm{m}$, because the other similar naphthenes such as cyclohexane $(50.0 \mathrm{mN} / \mathrm{m} \text { at } 298.15 \mathrm{~K})^{1}$ and decalin (cis-decalin, $51.24 \mathrm{mN} / \mathrm{m}$ at $288.15 \mathrm{~K}$ ) ${ }^{1}$ have interfacial tensions with water in this range. Moreover, saturated alkanes such as n-hexane and n-heptane also have interfacial tensions as $51.1 \mathrm{mN} / \mathrm{m}$ at $293.15 \mathrm{~K}$ and 50.2 at $298.15 \mathrm{~K}^{1}$, respectively. Also, Frietes et al. ${ }^{27}$ have predicted the value of methylcyclohexane as $50.8 \mathrm{mN} / \mathrm{m}$ at $293.15 \mathrm{~K}$ while Apostoluk and Drzymala ${ }^{22}$ have predicted the value equal to $49.61 \mathrm{mN} / \mathrm{m}$. As methylcyclohexane has only negligible solubility with water therefore the initial interfacial tension measured in this work may be regarded as the interfacial tension between the mutually saturated phases and should be a part of 
the interfacial tension database. For the case of cyclohexanol, it is important to mention that cyclohexanol has melting point of $299.08 \mathrm{~K}^{23}$ therefore the interfacial tension value of 3.92 $\mathrm{mN} / \mathrm{m}^{1}$ at $289.35 \mathrm{~K}$ is doubtful though mutual saturation with water may lead the possibility, it however, should be checked. Interfacial tension of diethyl ether-water may also need to be confirmed. For diethyl ether-water system, Backes et al. ${ }^{5}$ and Donahue and Bartell ${ }^{7}$ have found a value of $11.0 \mathrm{mN} / \mathrm{m}$ at $298.15 \mathrm{~K}$, Demond and Lindner ${ }^{1}$ have reported a value of $10.7 \mathrm{mN} / \mathrm{m}$ at 293.15 K, Santos et al. ${ }^{13}$ have also worked out a value of 10.7 but at $303.15 \mathrm{~K}$, and Kwok et al. ${ }^{11}$ measured a value of $7.36 \mathrm{mN} / \mathrm{m}$ at $298.15 \mathrm{~K}$ compared to initial interfacial tension of $9.03 \mathrm{mN} / \mathrm{m}$ at $293.15 \mathrm{~K}$ measured in the present study.

\section{Effect of composition on initial interfacial tension}

The effect of propionic acid concentration in aqueous solution on the initial interfacial tension for 4 water-organic systems is provided in Table 3 and graphically shown in Fig. 2. It is observed that varying the solute concentration in the aqueous phase significantly affects the initial interfacial tension and for all the cases of the experimentation, initial interfacial tension appears to decrease with an increase in the solute concentration. Initial interfacial tension without the solute (propanoic acid) in the system, i.e., for the binary organic-water system is observed as the maximum in all the cases. This may be due to the reason of decreased solubility of the components in water. As the propanoic acid concentration increases in a system the mutual solubility of the phases increases thereby decreases the interfacial surface (drop volume) as well as the initial interfacial tension. The extent and trend of decrease in initial interfacial tension is different in different systems depending may be on the initial interfacial tensions measured without the solute presence or on the basis of different miscibility characteristics of propanoic acid with an organic substance. Taking an example of toluene-water-propionic acid system, it is observed from Fig. 2 that the rate of decrease of initial interfacial tension is quite large initially as compared to the other systems and the rate decreases with an increase in propionic acid concentration and may eventually lead to the region where all the three components are miscible. The ternary initial interfacial data is fitted using the following exponential decay function (Eq. 5) and the values of the parameters so obtained are given in Table 4 along with the corresponding $\mathrm{R}^{2}$ values. In each case, the $\mathrm{t}$-value ( $\mathrm{t}$-value is a ratio of parameter value to its standard error) of the parameters in was found well above 2.0 .

$$
\gamma_{0}=a \exp \left(-b w_{H P r}\right)
$$

where, $a(\mathrm{mN} / \mathrm{m})$ and $b$ are constants and have to be obtained by fitting of the data and $w_{H P r}$ is weight percent of propionic acid in water. $\mathrm{Li}$ and $\mathrm{Fu}^{10}$ have also observed a decrease in 
interfacial tension for the increase in propionic acid concentration when measured between ternary phases of water + toluene + propionic acid.

\section{Effect of temperature on initial interfacial tension}

The effect of temperature on initial interfacial tension for five organic-water systems is shown in Table 5 and Fig. 3. It is observed that the values of the initial interfacial tension are only slightly affected by a change in temperature and the variation may be ignored in engineering calculations. This phenomenon of reduced effect of temperature on interfacial tension has also observed by other researchers in the field ${ }^{9,18,19}$.

\section{Conclusion}

This work reports initial interfacial tensions of 10 organic-water binary systems. The organic compounds include n-butyl acetate, cyclohexanol, cyclohexanone, diethyl ether, ethyl acetate, methyl ethyl ketone, methylcyclohexane, 1-octanol, and toluene. Our initial interfacial tensions are in agreement with the interfacial tensions measured in the literature for mutually saturated phases whenever possible. The literature value of the interfacial tension of methylcyclohexane is corrected and the measured initial interfacial tension is suggested to be included in the interfacial tension database. The effect of propionic acid concentration in water and temperature on initial interfacial tension of 4 organic-water system is measured. The solute addition has pronounced effect on the initial interfacial tensions while the effect of temperature is found only marginal. The method of initial interfacial tension measurement may not yield the exact value of interfacial tensions especially for relatively highly mutually soluble phases but it is a rapid mean of interfacial tension measurement close to the exact values as no mutual saturation is required till equilibration and the densities of the mutually saturated phases are difficult to find in most of the cases. The literature survey about the correlations for $F$ correction factor finds that the correlation complied by Drelich et al. ${ }^{30}$ is not universal but is applicable to a limited range of volume. 


\section{References}

(1) Demond, A. H.; Lindner, A. S. Estimation of Interfacial Tension Between Organic Liquids and Water. Environ. Sci. Techol. 1993, 27, 2318-2331.

(2) Khawaja, S. Y.; Usman, M. R.; Khan, S.; Afzal, W.; Akhtar, N. A. Dispersed Phase Holdup in a Tall and Low Plate Free Area Liquid Pulsed Sieve-Plate Extraction Column. Sep. Sci. Technol 2013, 48, 175-182.

(3) Lorenz, M.; Haverland, H.; Vogelphol, A. Fluid Dynamics of Pulsed Sieve Plate Extraction Columns. Chem. Eng. Technol. 1990, 13, 411-422.

(4) Pound, J. R. Interfacial Tensions Between Organic Liquids and Water or Aqueous Solutions. J. Phys. Chem. 1926, 30, 791-817.

(5) Backes, H. M.; Jun, M. J.; Bender, E.; Maurer, G. Interfacial Tensions in Binary and Ternary Liquid-Liquid Systems. Chem. Eng. Sci. 1990, 45, 275-286.

(6) Andreas, J. M.; Hauser, E. A.; Tucker, W. B. Boundary Tension by Pendant Drops. J. Phys. Chem 1938, 42, 1001-1019.

(7) Donahue, D. J.; Bartell, F. E. The Boundary Tension at Water-Organic Liquid Interfaces. J. Phys. Chem. 1952, 56, 480-484.

(8) Alpbaz, M.; Bilgesu, A.; Tutkun, O. The Measurement of Interfacial Tension by DropWeight Method. Commun. Fac. Sci. Univ. Ank. Series B 1988, 34, 103-112.

(9) Villers, D.; Platten, J. K. Temperature Dependence of the Interfacial Tension Between Water And Long-Chain Alcohols. J. Phys. Chem. 1988, 92, 4023-4024.

(10) Li, B.; Fu, J. Interfacial Tensions of Two-Liquid-Phase Ternary Systems. J. Chem. Eng. Data $1992,37,172-174$.

(11) Kwok, D. Y.; Hui, W.; Lin, R.; Neumann, A. W. Liquid-Fluid Interfacial Tensions Measured by Axisymmetric Drop Shape Analysis: Comparison Between the Pattern of Interfacial Tensions of Liquid-Liquid and Solid-Liquid Systems. Langmuir 1995, 11, 2669-2673.

(12) Mainzer, T.; Woermann, D. Temperature Dependence of Liquid-LiquidI Tension And Universal Critical Amplitude Ratio: An Experimental Study. Physica A 1996, 225, 312-322. 
(13) Santos, B. M. S.; Ferreira, A. G. M.; Fonseca, I. M. A. (2003). Surface and Interfacial Tensions of the Systems Water + n-Butyl Acetate + Methanol and Water + n-Pentyl Acetate + Methanol at 303.15 K. Fluid Phase Equilib. 2003, 208, 1-21.

(14) Kahl, H.; Wadewitz, T.; Winkelmann, J. Surface Tension and Interfacial Tension of Binary Organic Liquid Mixtures. J. Chem. Eng. Data 2003, 48, 1500-1507.

(15) Kijevcanin, M. L.; Ribeiro, I. S. A.; Ferreira, A. G. M.; Fonseca, I. M. A. Water + Esters + Methanol: Experimental Data, Correlation and Prediction of Surface and Interfacial Tensions at 303.15 K and Atmospheric Pressure. Fluid Phase Equilib. 2004, 218, 141-148.

(16) Johnson, I.; Costa, H. F.; Ferreira, A. G. M.; Fonseca, I. M. A. Density, Viscosity, And Surface and Interfacial Tensions of Mixtures of Water + n-Butyl Acetate+ 1-Propanol at 303.15 K And Atmospheric Pressure. Int. J. Thermophys. 2008, 29, 619-633.

(17) Costa, H. F.; Lourenço, H.; Johnson, I.; Gonçalves, F. A. M. M.; Ferreira, A. G. M.; Fonseca, I. M. A. Liquid- Liquid Equilibria, Density, Viscosity, and Surface and Interfacial Tension of the System Water + n-Butyl Acetate + 1-Propanol at $323.15 \mathrm{~K}$ and Atmospheric Pressure. J. Chem. Eng. Data, 2009, 54, 2845-2854.

(18) Zeppieri, S.; Rodríguez, J.; López de Ramos, A. L. Interfacial Tension of Alkane + Water Systems. J. Chem. Eng. Data 2001, 46, 1086-1088.

(19) Chavepeyer, G.; De Saedeleer, S.; Platten, J. K. Temperature Dependence of Interfacial Tension Between Normal Organic Acids and Water. J. Colloid Interface Sci. 1994, 167, 464-466.

(20) Murphy, N. F.; Lastovica, J. E.; Fallis, J. G. 1957. Correlation of Interfacial Tension of Two-Phase Three-Component Systems. Ind. Eng. Chem. 1957, 49, 1035-1042.

(21) Asadabadi, S.; Saien, J.; Khakizadeh, V. Interface Adsorption and Micelle Formation of Ionic Liquid 1-Hexyl-3-Methylimidazolium Chloride in the Toluene + Water System. J. Chem. Thermodyn. 2013, 62, 92-97.

(22) Cardenas, H.; Cartes, M.; Meija, A. Atmospheric Densities and Interfacial Tensions for 1Alkanol (1-Butanol to 1-Octanol) + Water and Ether (MTBE, ETBE, DIPE, TAME and THP) + Water Demixed Mixtures. Fluid Phase Equilib. 2015, 396, 88-97. 
(23) Tian, Y.; Cao, L.; Qiu, L.; Zhu, R. Comparison Study on Temperature Dependence of the Interfacial Tension of n-alkane Water and n-Alcohol Water Two Binary Systems. J. Chem. Eng. Data 2014, 59, 3495-3501.

(24) Harkins, W. D.; Cheng, Y. C. The Orientation of Molecules in Surfaces. VI. Cohesion, Adhesion, Tensile Strength, Tensile Energy, Negative Surface Energy, Interfacial Tension, and Molecular Attraction. J. Am. Chem. Soc. 1921, 43, 35-53.

(25) Carrera, G. V. S. M.; Alfonso, C. A. M.; Branco, L. C. Interfacial Properties, Densities, and Contact Angles of Task Specific Ionic Liquids. J. Chem. Eng. Data 2010, 55, 609-615.

(26) Pliskin, J.; Treybal, R. E. Interfacial Tensions of Binary and Ternary Two-Phase Liquid Systems. AIChE J. 1966, 12, 795-801.

(27) Freitas, A. A.; Quina, F. H.; Carroll, F. A. Estimation of Water-Organic Interfacial Tensions. A Linear Free Energy Relationship Analysis of Interfacial Adhesion. J. Phys. Chem. B 1997, 101, 7488-7493.

(28) Evans, M. J. B. Measurement of Surface and Interfacial Tension. In: Experimental Thermodynamics, Measurement of the Thermodynamic Properties of Multiple Phases, Weir, R. D.; de Loos, T. W. (Eds.), Elsevier: Amsterdam, 2005.

(29) Berg, J. C. An Introduction to Interface and Colloids: The Bridge to Nanoscience; World Scientific Publishing Co.: London, 2010.

(30) Drelich, J.; Fang, C.; White, C. L. Measurement of Interfacial Tension in Fluid-Fluid Systems. In: Encyclopedia of Surface And Colloid Science; Vol. 3, Hubbard, A. T. (Ed.), Marcel Dekker, Inc.: New York, 2002.

(31) Apostoluk, W.; Drzymała, J. An Improved Estimation of Water-Organic Liquid Interfacial Tension Based on Linear Solvation Energy Relationship Approach. J. Colloid Interface Sci. 2003, 262, 483-488.

(32) Lide, D. R., Ed. CRC Handbook of Chemistry and Physics. $87^{\text {th }}$ ed., Taylor and Francis: Boca Raton, 2007. 


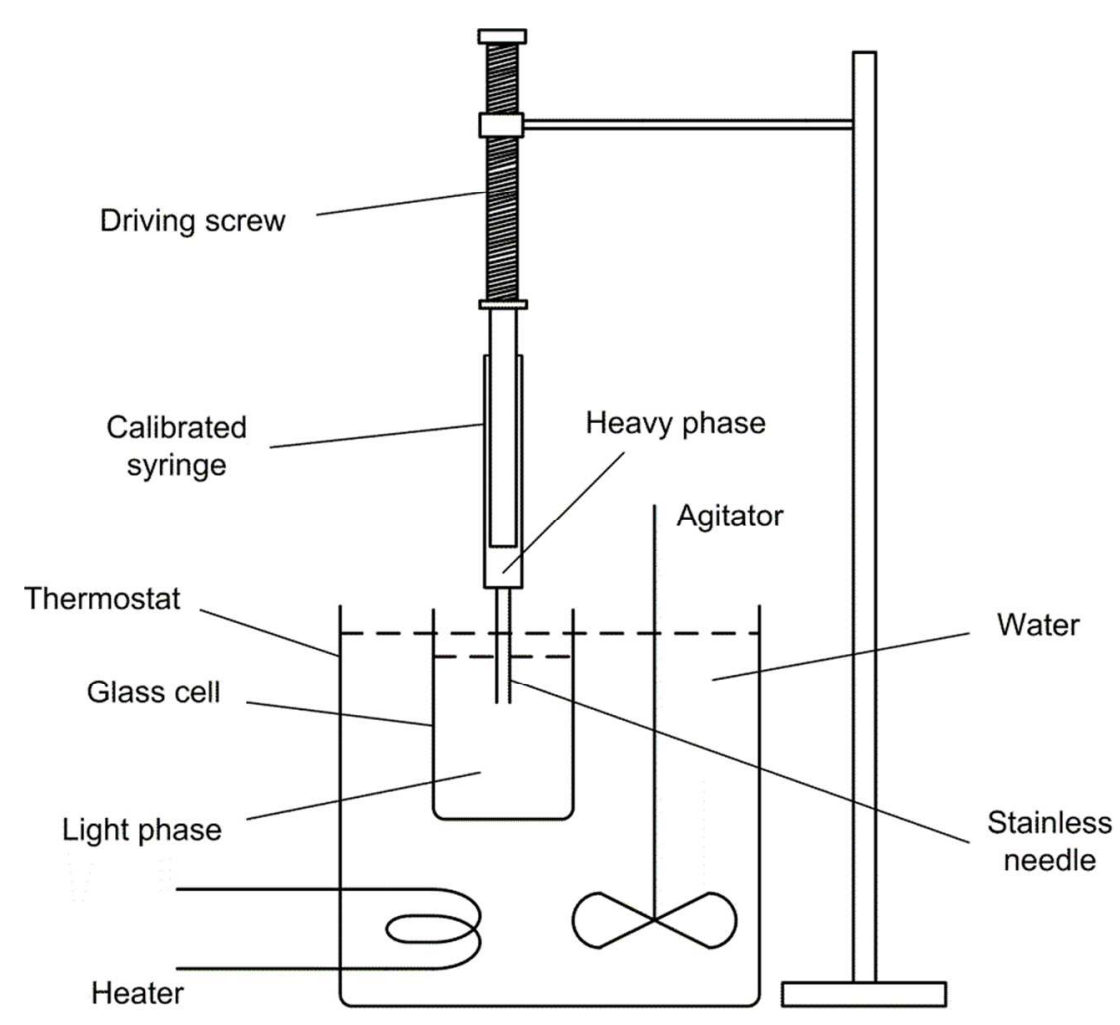

Fig. 1 Experimental setup (not to scale). 
1

2

3

4

5

6

7

8

9

10

11

12

13

14

15

16

17

18

19

20

21

22

23

24

25

26

27

28

29

30

31

32

33

34

35

36

37

38

39

40

41

42

43

44

45

46

47

48

49

50

51

52

53

54

55

56

57

58

59

60

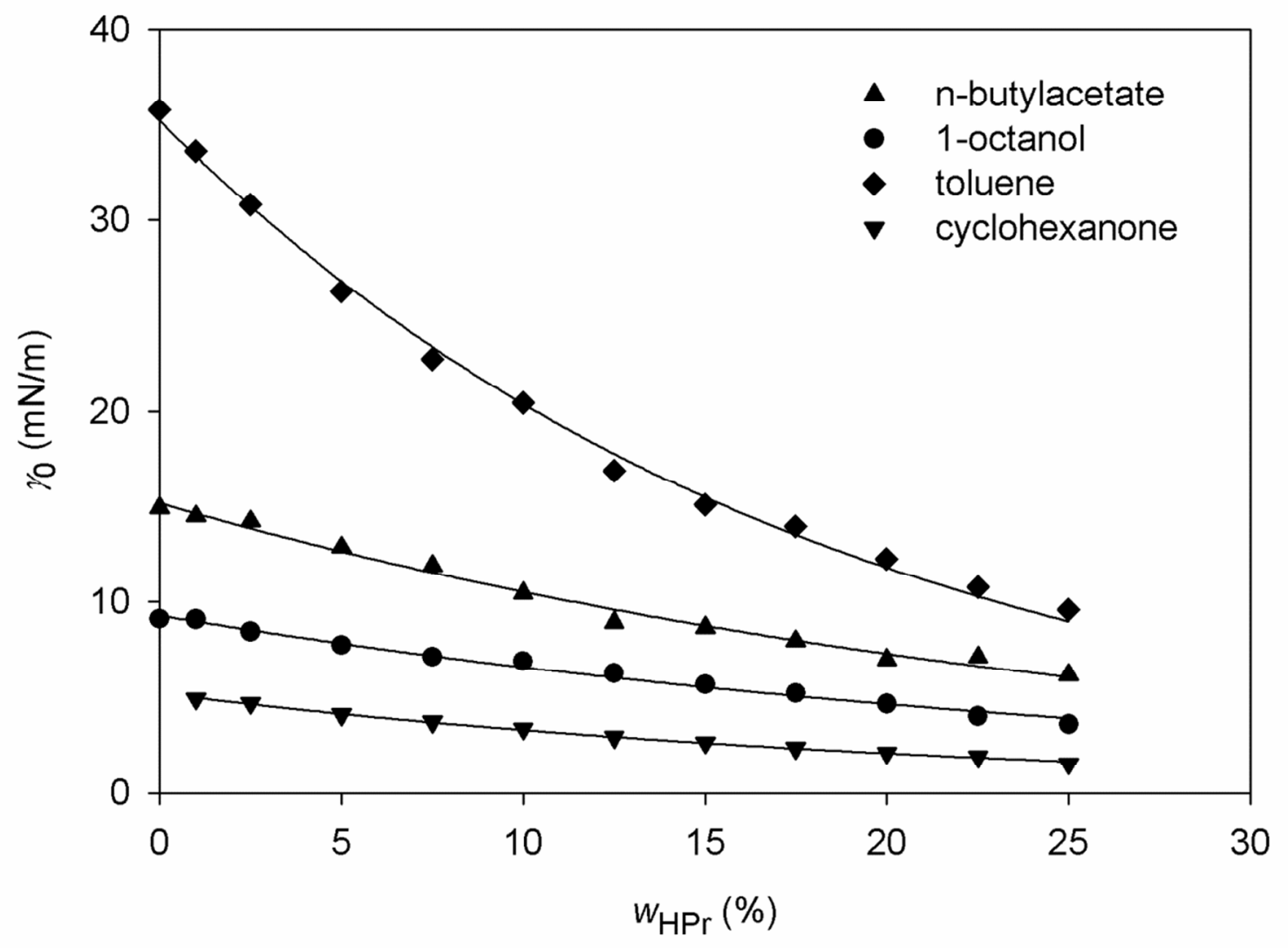

Fig. 2 Effect of propionic acid concentration on the initial interfacial tension of various waterorganic systems. Solid lines show the model results given by Eq. 5 . 


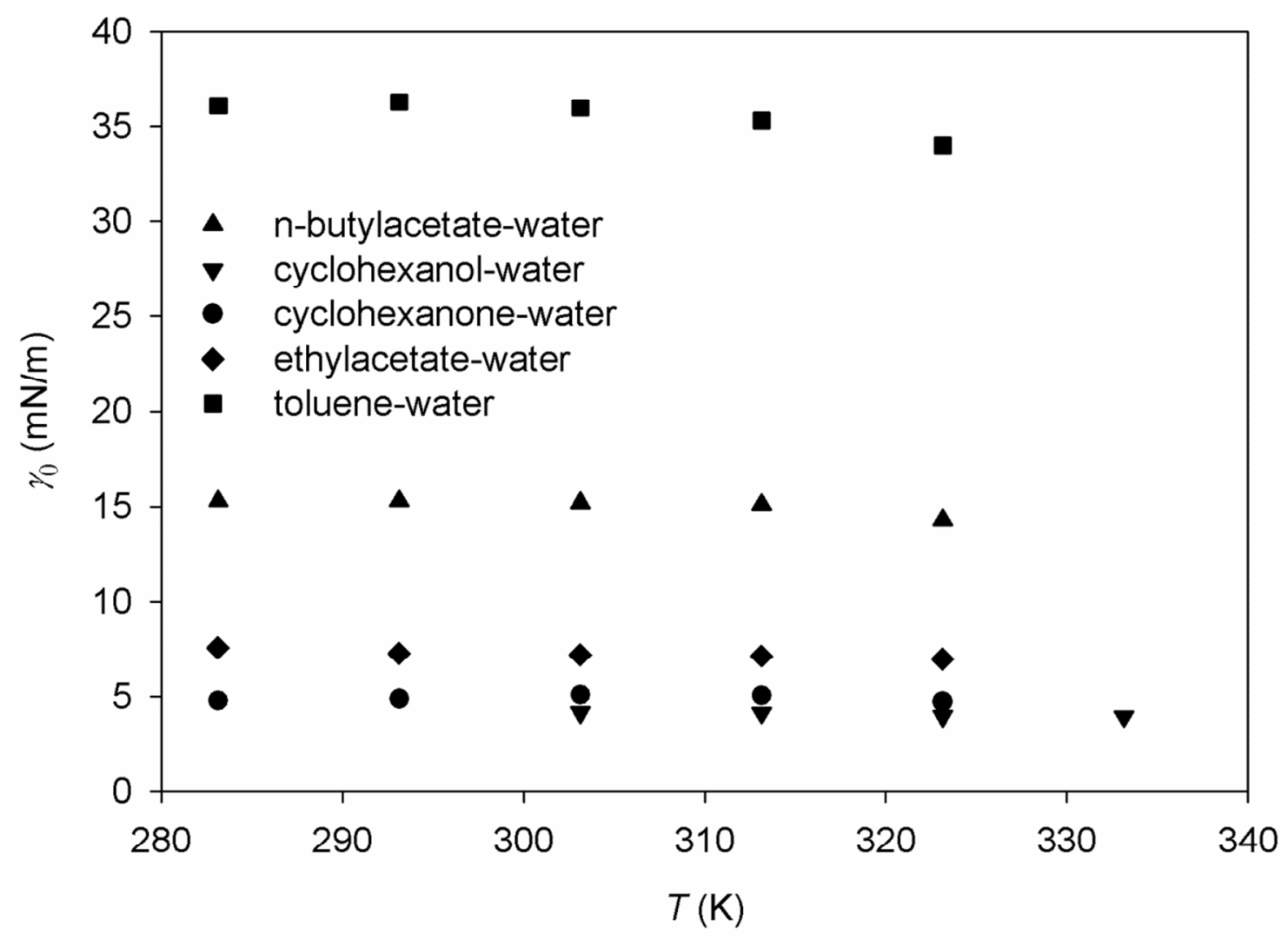

Fig. 3 Effect of temperature on the initial interfacial tension of various water-organic systems. 
Table 1 Details of the chemicals used in the present study

\begin{tabular}{lccccc}
\hline Product & $\begin{array}{c}\mathrm{CAS} \\
\text { Number }\end{array}$ & $\begin{array}{c}\text { Chemical } \\
\text { formula }\end{array}$ & $\begin{array}{c}\text { Purity } \\
(\mathrm{wt} \%)\end{array}$ & $\begin{array}{c}\text { Water content } \\
(\mathrm{wt} \%)\end{array}$ & Supplier \\
\hline n-butyl acetate & $123-86-4$ & $\mathrm{C}_{6} \mathrm{H}_{12} \mathrm{O}_{2}$ & 99 & 0.05 & Lab-Scan \\
cyclohexanol & $108-93-0$ & $\mathrm{C}_{6} \mathrm{H}_{12} \mathrm{O}$ & $\geq 98$ & - & Fluka Chemika \\
cyclohexanone & $108-94-1$ & $\mathrm{C}_{6} \mathrm{H}_{10} \mathrm{O}$ & $>99$ & $<0.2$ & Merck \\
diethyl ether & $60-29-7$ & $\mathrm{C}_{4} \mathrm{H}_{10} \mathrm{O}$ & $\geq 99.5$ & $\leq 0.2$ & Riedel-de Haën \\
ethyl acetate & $141-78-6$ & $\mathrm{C}_{4} \mathrm{H}_{8} \mathrm{O}_{2}$ & $>99$ & $<0.1$ & Merck \\
methyl cyclohexane & $108-87-2$ & $\mathrm{C}_{7} \mathrm{H}_{14}$ & $\geq 99$ & anhydrous & Sigma-Aldrich \\
methyl ethyl ketone & $78-93-3$ & $\mathrm{C}_{4} \mathrm{H}_{8} \mathrm{O}$ & 99.5 & 0.1 & Lab-Scan \\
1-octanol & $111-87-5$ & $\mathrm{C}_{8} \mathrm{H}_{18} \mathrm{O}$ & 99 & 0.1 & Panreac Sintesis \\
toluene & $108-88-3$ & $\mathrm{C}_{7} \mathrm{H}_{8}$ & $\geq 99.5$ & $\leq 0.05$ & Riedel-de Haën \\
water, de-ionized & $7732-18-5$ & $\mathrm{H}_{2} \mathrm{O}$ & 100 & 100 & Local market \\
\hline
\end{tabular}

Table 2 Experimental initial interfacial tension of various organic-water systems at ambient pressure $(101 \mathrm{kPa})$

\begin{tabular}{|c|c|c|c|c|c|c|}
\hline System & $\begin{array}{c}\text { This } \\
\text { work } \\
\gamma_{0} \\
(\mathrm{mN} / \mathrm{m})^{a}\end{array}$ & $\begin{array}{c}T \\
(\mathrm{~K})\end{array}$ & $\begin{array}{l}\text { Literature } \\
\gamma(\mathrm{mN} / \mathrm{m})\end{array}$ & $\begin{array}{c}T \\
(\mathrm{~K})\end{array}$ & $\begin{array}{c}S^{32} \\
\mathrm{~g} / \mathrm{L}-\mathrm{H}_{2} \mathrm{O}\end{array}$ & $\begin{array}{c}T \\
(\mathrm{~K})\end{array}$ \\
\hline n-butyl acetate & 14.95 & 290.15 & $14.5^{7}$ & 298.15 & 6.80 & 293.15 \\
\hline cyclohexanol & 4.17 & 303.15 & $3.7^{5}$ & 298.35 & 40.0 & 298.15 \\
\hline cyclohexanone & 4.87 & 293.15 & $3.9^{5}$ & 293.85 & 96.0 & 298.15 \\
\hline diethyl ether & 9.03 & 293.15 & $\begin{array}{l}10.70^{1} \\
7.36^{11}\end{array}$ & $\begin{array}{l}293.15 \\
298.15\end{array}$ & 64.2 & 298.15 \\
\hline ethyl acetate & 7.37 & 289.15 & $6.8^{1}$ & 298.15 & 87.9 & 298.15 \\
\hline methyl ethyl ketone & $0.92^{b}$ & 292.15 & $1.0^{20}$ & 298.15 & $\sim 210$ & 298.15 \\
\hline methylcyclohexane & 51.40 & 293.15 & $41.9^{5}$ & 298.15 & 0.015 & 298.15 \\
\hline 1-octanol & 9.10 & 289.15 & $8.52^{1}$ & 293.15 & 0.540 & 298.15 \\
\hline Toluene & 35.80 & 290.15 & $35.4^{5}$ & 298.15 & 0.531 & 298.15 \\
\hline
\end{tabular}

${ }^{a}$ Standard uncertainties are $u(T)=0.25 \mathrm{~K}, u(V)=0.01 \mathrm{~cm}^{3}$. Expanded uncertainty for the initial interfacial tension $\left(\gamma_{0}\right)$ is $U(c)=0.50 \mathrm{mN} / \mathrm{m}\left(0.95\right.$ level of confidence). ${ }^{b}$ only measured for once. $\gamma_{0}$, initial interfacial tension; $\gamma$, interfacial tension (of mutually saturated phases); $s$, solubility of organic component in water; $T$, temperature of measurement. 
Table 3 Effect of propionic acid concentration in water on the initial interfacial tension of various organic-water systems at ambient pressure $(101 \mathrm{kPa})$

\begin{tabular}{cccccc}
\hline \multirow{2}{*}{$\begin{array}{c}w_{H P r} \\
(\mathrm{wt} \%)\end{array}$} & $x_{H P r}$ & \multicolumn{5}{c}{$\gamma_{0}(\mathrm{mN} / \mathrm{m})^{a}$} \\
\cline { 3 - 6 } & & n-butyl acetate & cyclohexanone & 1-octanol & toluene \\
\cline { 3 - 6 } & & $T=289.15 \mathrm{~K}$ & $T=289.15 \mathrm{~K}$ & $T=289.15 \mathrm{~K}$ & $T=290.15 \mathrm{~K}$ \\
\hline 0.0 & 0.0 & 14.9 & - & 9.10 & 35.8 \\
1.0 & 0.0025 & 14.5 & 4.92 & 9.08 & 33.6 \\
2.5 & 0.0062 & 14.2 & 4.70 & 8.43 & 30.8 \\
5.0 & 0.0126 & 12.9 & 4.10 & 7.73 & 26.3 \\
7.5 & 0.0193 & 11.9 & 3.73 & 7.12 & 22.8 \\
10.0 & 0.0263 & 10.5 & 3.35 & 6.90 & 20.4 \\
12.5 & 0.0336 & 8.92 & 2.92 & 6.26 & 16.9 \\
15.0 & 0.0412 & 8.66 & 2.63 & 5.67 & 15.1 \\
17.5 & 0.0491 & 7.95 & 2.35 & 5.20 & 13.9 \\
20.0 & 0.0573 & 6.99 & 2.11 & 4.67 & 12.2 \\
22.5 & 0.0660 & 7.12 & 1.89 & 4.02 & 10.7 \\
25.0 & 0.0750 & 6.18 & 1.53 & 3.59 & 9.57 \\
\hline
\end{tabular}

${ }^{a}$ Standard uncertainties are $u(x)=0.0001, u(T)=0.25 \mathrm{~K}, u(V)=0.01 \mathrm{~cm}^{3}$. Expanded uncertainty for the initial interfacial tension $\left(\gamma_{0}\right)$ is $U(c)=0.50 \mathrm{mN} / \mathrm{m}(0.95$ level of confidence $) . x_{H P r}$ is mole fraction of propionic acid in water.

Table 4 Parameters of Eq. 5 fitted for ternary propionic acid- organic solvent-water systems

\begin{tabular}{ccccc}
\hline & \multicolumn{4}{c}{ Parameter values } \\
\cline { 2 - 5 } & n-butyl acetate & cyclohexanone & 1-octanol & toluene \\
\hline$a(\mathrm{mN} / \mathrm{m})$ & 15.1977 & 4.9480 & 9.2911 & 35.2570 \\
$b$ & 0.0368 & 0.0421 & 0.0346 & 0.0548 \\
$\mathrm{R}^{2}$ & 0.9892 & 0.9975 & 0.9892 & 0.9967 \\
\hline
\end{tabular}

Table 5 Effect of temperature on the initial interfacial tension of various water-organic systems at ambient pressure $(101 \mathrm{kPa})$

\begin{tabular}{cccccc}
\hline$T(\mathrm{~K})$ & n-butyl acetate & cyclohexanol & cyclohexanone & ethyl acetate & toluene \\
\cline { 2 - 5 } & \multicolumn{5}{c}{$\gamma_{0}(\mathrm{mN} / \mathrm{m})^{a}$} \\
\hline 283.15 & 15.3 & - & $4.78^{b}$ & 7.61 & 36.1 \\
293.15 & 15.3 & - & 4.87 & 7.29 & 36.3 \\
303.15 & 15.2 & 4.17 & 5.08 & 7.19 & 36.0 \\
313.15 & 15.1 & 4.15 & 5.05 & 7.15 & 35.3 \\
323.15 & 14.3 & 3.97 & 4.71 & 6.99 & - \\
333.15 & - & 3.95 & - & - & - \\
\hline${ }^{a}$ Standard uncertainties are $u(T)=0.25 \mathrm{~K}, u(V)=0.01 \mathrm{~cm}^{3}$. Expanded uncertainty for the initial \\
interfacial tension $\left(\gamma_{0}\right)$ is $U(c)=0.50 \mathrm{mN} / \mathrm{m}\left(0.95\right.$ level of confidence). ${ }^{b}$ Only measured for once.
\end{tabular}




\section{TOC Graphic}

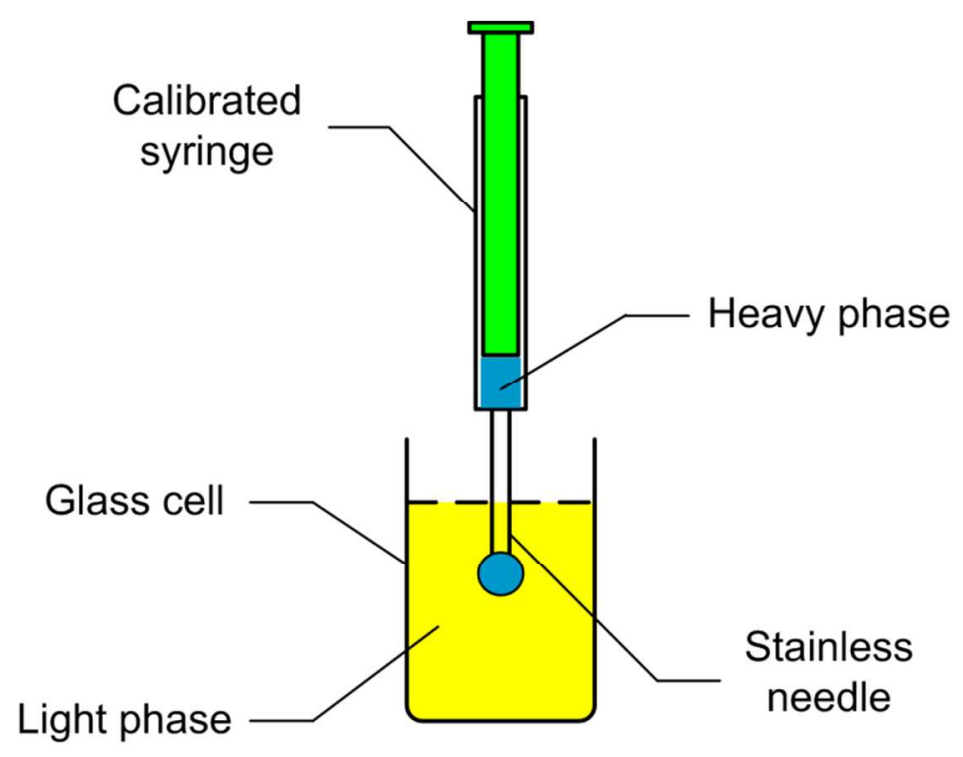

25

26

27

28

30

31

32

33

34

35

37

38

39

40

41

42

43
44

45

46

47

48

49

51

52

53

54

55

57

58

59

60 